\title{
SEARCH ENGINE OPTIMIZATION
}

\author{
Deepthi.K $\mathbf{K}^{1}$ \\ ${ }^{1}$ Assistant Professor, Department of Computer Science, College of Engineering, Vadakara, Kozhikkode, Kerala
}

\begin{abstract}
If anyone wants to get better rank for their website in search engine then there is a necessity to do SEARCH ENGINE OPTIMIZATION (SEO). SEO is a very powerful web marketing technique. It helps to increase website's rank as well as traffic from search engines. With a deeper knowledge of SEARCH ENGINE OPTIMIZATION best practices, organizations can avoid unethical practices and effectively utilize strategies approved by popular search engines. SEO can be proved very beneficial for small business as well as for giant business. This paper focuses on important ideas associated with Search Engine Optimization. Paper discusses the optimization techniques using. keywords, title, alt, metatags, anchor, and other text.
\end{abstract}

Keywords: SEO, Web Marketing Technique, Giant Business, Optimization Techniquies.

$* * *$

\section{INTRODUCTION}

A web search engine is a software system that is designed to search for information on the World Wide Web .Webmasters started optimizing sites from the view point of SEO in 1990s. Site owners started recognizing the value of getting their sites high rank and visibility in search engine results.

First search engine algorithms were based on information provided in metatag or index files. Metatags gives a short description about page content. But relying on metatags was less reliable as the given information in metatag may not be relevant to the page. Giving fake data in metatags helped to rank incomplete and irrelevant sites higher in search results. Web developers used a number of fake attributes in HTML source of a page to get high rank in search engines.

Google was founded by Page and Brin in 1998.Google became very popular due to its simpler structure. Google used off-page factors such as PageRank and hyperlink analysis and on-page factors for their ranking. Numerous sites concentrated on exchanging, buying, and selling links. Some of these schemes, or link farms, developed thousands of sites for the purpose of link spamming. Search engines used a huge range of undisclosed factors, for reducing the impact of link schemes. Google tells it uses 200+ signals to rank sites. The leading search engines, Google, Yahoo, Live Search etc do not reveal the algorithms they use to rank pages.

\section{SEARCH ENGINE OPTIMIZATION}

SEO is a technique for:

- Designing and developing a website to rank well in search engine results.

- Improving the volume and quality of traffic to a website from search engines.

- Marketing by understanding how search algorithms work.

It is guaranteed that, a top ranking in the SERP (Search Engine Result Page) considerably boosts organization's visibility. For achieving a high SERP ranking, webmasters use some practices, which is known as search-engine optimization (SEO). For good ranking current SEO practices are surveyed and examined possible future directions. This paper describes a list of tools and methods to help organizations that are new to SEO. These resources help web developers to develop websites that exploits procedures used by popular search engines.

\subsection{Search Engine Basics}

- Processing queries: When a data or query is entered in the search engine's tool bar, the engine search its index and list all the documents which match the data.

- Classifying results: When the engine discovered the matching results, its algorithm uses some calculations to rank the results in the order of relevancy.

\subsection{Working}

\subsubsection{Basics}

When you perform a search in the search engine's tool bar, $u$ will get a set of results from all over the web. When you do a search, the search engine checks its index and give you the most appropriate search results.

The main procedures in giving search results are:

a) Crawling: "Spiders" are automated programs used by search engines for crawling. Using the hyperlink structure, spiders "crawl" the pages in the World Wide Web. Among the approximate 30 billion pages, search engines usually crawls be 7 to 10 billion pages.

b) Indexing: search engines crawls the web page , "index" its contents and load the contents in a huge database. Management of this index is of prime importance as all the requests which search for a document have to search trillions of documents in fraction of second.

c) Processing Queries: When a user requests a data in search engine, it fetch all the matching data from its index .When the specified word or phrase is found in the web page a match is said to occur. 
d) Ranking Results: When a match is discovered, the algorithm determines the most relevant pages through some calculations. The result is displayed in the order of most to least relevancy.

e) Serving results: When a user searches for a query, the engine search the index for matching pages and return the results which are most relevant to the user.

\section{ALGORITHMS}

Algorithms are computer programs that search clues and provide you what you want. Since the web contains trillions of web pages, there will be thousands of pages which have useful information.

Google's algorithms now use 200+ signals for answering users query. These signals are very important for a marketer. But google does not give out details about these signals. These signals decide the rank of the website in search results. For example, these signals can be keywords on websites, freshness of site content, the ranking of the page in internet search etc.

Search engines crawls and index web pages for calculating ranks. Each search engine has their own crawling robot. The crawling robot of Google is known as "googlebot". For determining whether a site is relevant or not, the crawling robots analyze each page's contents by moving from page to page.

\subsection{Google Algorithms Major Changes}

Google developed the search algorithm known as PageRank. To find out the importance of the website, this algorithm checks the number and quality of links on each page. It is assumed that, a relevant website will have more links from other websites.

Google developed more algorithms named Google Panda, an algorithm focuses on removing low quality websites and Google Penguin, an algorithm that reduces spam websites.

1) Pagerank: This algorithm is used by Google to rank websites in their search results. PageRank is used to rank websites according to their relevancy. It determines the importance of web pages based on link structure. PageRank algorithms assign numerical weightings to hyperlinked documents indexed by a search engine. The Google search engine uses pagerank algorithm to determine a page's relevance or importance. In fact pagerank is a "rank", given by all other pages on the web, based on the importance of the web page. A link to a page is counted as a poll of support.

2) Google Panda: This algorithm was developed in 2011, and it affected many sites. Its aim was to detect sites with low quality contents. Sites with low quality contents are known as "content farms". Now panda is considered as the core ranking algorithm of Google. Panda is Google's most important spam-fighting algorithms.
Panda algorithm removes sites with low-quality which give lesser information. This algorithm gives higher ranks for sites having original and quality contents. Major changes in search results occurred due to this algorithm and it made lot of discussion. This was the aim of Google. Panda is updated from time-to-time. It is beneficial to previously hit sites which made right changes.

3) Google Penguin: Google's next algorithm was named as Penguin. Penguin was launched in April 2012 and its main aim was to eliminate web spam. Ranking of sites which violate Google's quality principles is decreased by this algorithm. These violations are mainly cloaking, keyword stuffing, and duplicating the content purposefully.

Keyword stuffing is a method of using the keywords excessively in pages to increase the site's ranking. Using so many keywords in pages is a negative experience for the site user.

"Link schemes" are also considered as breaking the principles of Google's webmaster. Selling or buying links is considered as link scheming. Link exchanges and paid linking activity is not recommended for good search engine rankings. According to google, maintaining good contents in web pages bring relevant links to your pages naturally.

In short, exchanging links with friends may not always be a good practice. When sharing links it should be remembered that excessive link exchanges are not encouraged by Google.

4) Google Hummingbird: Another algorithm google started using recently is humming bird. Google launched Hummingbird on August $30^{\text {th }}$ of 2013. It was a major change in Google's history. Humming bird is designed to concentrate on the meaning behind the words. The name aims to humming birds which are "precise and fast".

Hummingbirds uses semantic search. That is they judge the context of a search. They consider the query as a whole rather than each word in the query. This results in the high ranking of matching pages, instead of pages containing few words.

\section{PAGE OPTIMIZATION}

\subsection{On Page SEO}

By On Page SEO, it is meant the whole procedures we do in our page to improve rank and visibility of the website. Onpage means the content and HTML source code of a page that can be optimized, in contradiction to off-page SEO which refers to links and other external signals. On Page Optimization optimizes your website to rank well in search engines. There will be high increase in the rank of the websites, when appropriate On Page optimization is done.

1) Title Optimization: It is a better practice to include a TITLE tag,inside the HTML head tag. By title tag it is meant what is displayed in the title bar of the web page.Title tag is also displayed when a page is bookmarked or when it is 
added to our Favorites. The title tag is different from the heading for a page. For good ranking in search engines, there should only be one <title> tag used per page, and it should only be used within the <head $>$ section of the code.

This is the one place on a webpage where your keywords MUST be present. Correct use of keywords in the title of every page of your website is extremely important to Google - particularly for the homepage. If you do nothing else to optimize your site, remember to do this.

$\langle$ head $>$

$<$ title>

SEO India- search engine optimization India, seo services, seo company India, affordable seo India

$</$ title $>$

$</$ head $>$

Search engine optimization services
About $46,100,000$ results ( 0.17 seconds)

\section{SEO India - search engine optimization Www. softsolutionsindia.net/ - Cached SEO India, SEO Company India provides afforc India, organic seo, article writing, social bookm Contact us - Industrial Training - Resources - C}

Fig 1: Figure showing Title Optimization

2) Body Text: Contents of the web page are considered as a key factor for ranking in search engines. So website developers should concentrate on the contents when designing web pages.

Following are the key points to optimize body text:

- Use of heading tags.

- Word frequency: Average pages on a web page should be in the range 500 to 800 .

- Density of keywords: On a page containing 400-600 words, use of key words should be in between $2 \%-4 \%$ $\& 7 \%-9 \%$ on page containing $800+$ words.

- Relevant keywords: Most frequent keywords of the users request should be kept on top of the page. The web crawler considers the key word coming on top of a page as most significant for indexing.

- Meta Tags optimization: Meta tags give the search engine proper information about a website. Description tag is the most important tag among the different meta tags because it is a part of search result.If the searched phrase is contained in the description, search engines show the meta description in search results. Optimization of meta description is extremely important for on-page SEO.

3) Hyperlink: A hyperlink is a link from a hypertext document to another location. There should be a crawlable link structure in each page,for accessing all the pages on a web site. Some websites hide their main link navigation such that it cannot be accessed by search engines. This prevents the pages from listing in the engine's indices.Following figure illustrates this problem.

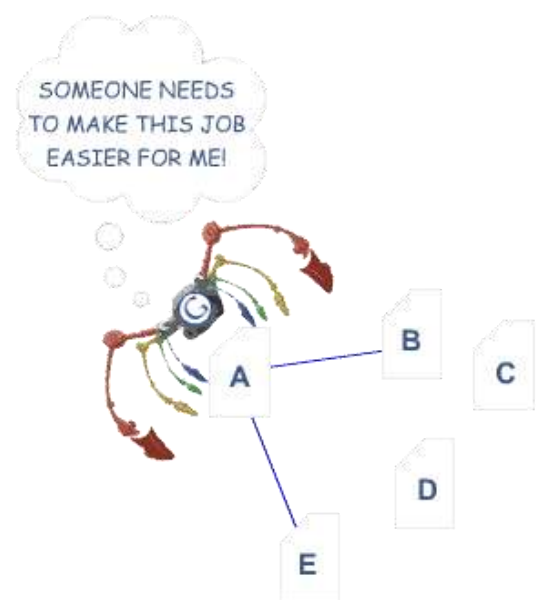

Fig 2: Figure showing Hyperlinks

Here, Google's spider reached page " $\mathrm{A}$ " and sees links to pages "B" and "E." But pages $C$ and D cannot be reached by the spider as there is no direct crawlable links present to those pages. An optimal website structure should be like the following (The big circle on top indicates the home page):

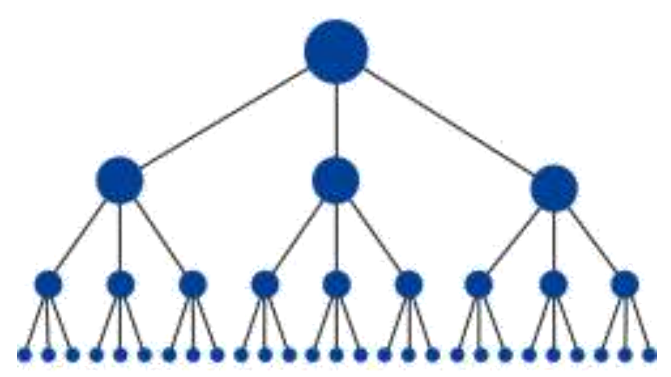

Fig 3: Figure showing optimal link structure

4) Image Optimization: For good image search optimization we should use the targeted keyword to name the image. Search engines will not understand what a picture is by scanning it. So they search for clues they look for clues in two places.

Each uploaded image in the web page will be having a file name. The image's name will be there in the source code of the web page. You have to use file names which narrate the picture. Because search engines will scan the source code of the site for clues. For example, "blue-flower.jpge is better than "image523.jpge.

Another improvement you can do is to use an alt tag with each image on your website. Alt tags are tags which allow you to add a short description with each image.

5) Sitemaps: We can make a sitemap in our website describing the pages. We have to make 2 sitemaps, for customers and for search engines. It will make the traversal easy both for users and search engines. 


\subsection{Off-Page SEO}

Off page SEO means the procedures that can be used to improve the rank of a web site in the search engine results page (SERPs). Its main objective is to concentrate on creating back links \& social media marketing. In short SEO implements numerous link building methods such as Social networking, Video submission, Blog posting, link exchange etc.

1) Backlink Generation: It is the process of increasing the links of our website in other websites. When a page has more backlinks, search engines assume there is something relevant in the page. Increasing backlinks will increase the rank of a web site. One way to do it is to provide the URL in online advertisements.

2) Blog Posting: Being on a blog like log spot, word press, thoughts, link orbits etc is beneficial. Companies having blogs have more visitors, more indexed pages and more inbound links.

3) Social Networking: Social networking helps for better brand visibility. It is the practice that improves sharing of contents, data exchange, unique content adding etc.

Following are the benefits of social networking sites:

- It improves website traffic

- It promotes visibility of a brand

- It creates inbound links

4) Video Submission: Search engine optimization can be increased in several ways using videos. Some of the techniques for video optimization is given below:

- Use a good title for the video, that is relevant for your product or service.

- Create a full description of the video. Emphasize key points from the video and it makes easy for the search engine to find the value this video offers.

- Use relevant content on the page around your videos.

- Use key phrases which describes the content for tagging videos.

- Use logo in the video so that it create brand awareness in users.

- Use the "Embed Option" in videos when uploading since it allows users to post the video in their sites/blogs.

\section{CONCLUSION}

Search engine optimization is the most appropriate practice to pull the customers to a website. It help the site to rank at high level when a search is made. The various methods discussed in the paper help web developers and owners to improve their site's visibility. Applying all these techniques effectively will boost the use of website and correct return for investment will be received for the owner. Search engine optimization is an effective practice which should be followed by all web designers and developers. Even though ranking in search engine organic results are unpredictable appropriate use of optimization techniques will help much. Since the use of search engines like Google, Yahoo!, Bing etc are so common today it is important to design every page following good optimization principles.

\section{REFERENCES}

[1]. Venkat N. Gudivada and Dhana Rao,. ," Understanding Search-Engine Optimization", IEEE Professional Communication

[2]. MengCui , SongyunHu, "Search Engine Optimization Research for Website Promotion" Information Technology, Computer Engineering and Management Sciences (ICM), 2011 International Conference on Volume:4

[3]. Zhang, Huanjiong, "Research a New Method of Search Engine Optimization", Internet Technology and Applications, 2010 International Conference

[4]. ZhouHui, QinShigang, LiuJinhua , Chen Jianli, "Study on Website Search Engine Optimization" Computer Science \& Service System (CSSS), 2012 International Conference [5]. Duk, S., Bjelobrk, D., Carapina, M., "SEO in ecommerce: Balancing between white and black hat methods"

[6]. Mo Yunfeng, "A Study on Tactics for Corporate Website Development Aiming at Search Engine Optimization" Education Technology and Computer Science (ETCS), 2010 Second International Workshop on Volume: 3 